\title{
Toxicidad y tratamientos de soporte en oncología radioterápica. Toxicidad en el área de O.R.L. Prevención y tratamiento
}

\author{
F. Mendicote León
}

\section{Introducción}

La irradiación de los tejidos del área de cabeza y cuello produce efectos tóxicos agudos, durante el tratamiento y en las semanas posteriores y tardíos, meses o años después.

Los tejidos de proliferación celular rápida, como la piel y las mucosas, presentan mayor afectación aguda. Los de proliferación celular lenta, p.e. tejido conectivo, óseo y tejido nervioso muestran su daño a largo plazo.

Entre los efectos agudos son habituales el eritema, la hiperpigmentación, ulceración de la piel, alopecia, mucositis, disgeusia, disfagia, disfonía, xerostomía, sialoadenitis, conjuntivitis y otitis.

Son efectos tardíos la fibrosis, necrosis, xerostomía, estenosis faríngea, trismus, catarata, queratitis corneal, retinopatía, neuritis óptica, edema submentoniano, hipotiroidismo, hipopituitarismo, mielitis y la pérdida dental.

La mayoría de los pacientes superan la toxicidad aguda a las 4-6 semanas de finalizar el tratamiento, salvo la xerostomía, que suele ser definitiva en mayor o menor grado. El edema laríngeo, la disgeusia, la hipoacusia y la disfagia pueden prolongarse durante más tiempo.

En general, la severidad de los efectos agudos no predice el riesgo de efectos tardíos, salvo cuando aquellos son extremadamente severos. Es importante conocer las dosis de tolerancia de los tejidos normales incluidos en el campo de tratamiento radioterápico para conseguir la curación con la menor toxicidad posible. Influyen en el riesgo de toxicidad la dosis total, dosis por sesión, intervalo entre las sesiones, homogeneidad de la dosis, tipo y calidad de la radiación, radiosensibilidad intrínseca del tejido y la administración de quimioterapia concomitante. Grandes inhomogeneidades en el volumen de tratamiento provocan áreas de sobredosificación recibiendo, así los tejidos, mayores dosis en cada se-

Instituto Oncológico de Castilla-La Mancha Ciudad Real sión, aumentando la dosis equivalente biológica y el riesgo de efectos tóxicos tardíos ${ }^{1}$.

\section{Mucositis}

El daño de la mucosa orofaríngea produce una serie de problemas que pueden impedir completar el tratamiento planificado sin interrupciones, con menor probabilidad de control tumoral. El dolor impide al paciente mantener un estado nutricional adecuado, con deterioro del estado general.

Ocasionada por el daño de las células basales de las mucosas $^{2}$, aparece a las 2 semanas de iniciarse el tratamiento y progresa de forma parcheada hasta hacerse confluente en los casos severos. Estos llegan al 50\% con radioterapia única o al $90 \%$ con quimioterapia concomitante ${ }^{3}$, por lo que es esencial la colocación de sonda de gastrostomía percutanea endoscópica para una correcta nutrición, hidratación y administración medicamentosa durante los tratamientos quimiorradioterápicos en el área de cabeza y cuello.

Para su prevención se recomienda una meticulosa valoración previa al tratamiento y correcta higiene bucodental ${ }^{4}$, aunque las soluciones que mezclan antibióticos y antifúngicos no han demostrado eficacia ${ }^{5}$; los enjuagues con agua salina bicarbonatada alivian más que el agua oxigenada diluida que puede ser perjudicial ${ }^{4}$; el agua de manzanilla parece ser útil en un estudio no controlado(6). El sulfato de zinc, agente cicatrizante que estimula el crecimiento del epitelio y neutraliza los radicales libres hidroxilo, a una dosis de 50 mgrs/8h consiguió reducir la severidad de la mucositis en un ensayo aleatorio y prospectivo 7 .

La bencidamina tiene propiedades antiinflamatorias, analgésicas, anestésicas y antimicrobianas. Como solución oral (Tantum Verde ${ }^{\circledR}$ ) es eficaz para reducir el dolor y severidad de la mucositis, aunque a dosis de radioterapia en torno a $50 \mathrm{~Gy}$, como demuestran ensayos aleatorizados 8 .

El factor de crecimiento de los queratinocitos recombinante humano (palifermin) ha demostrado su eficacia en reducir la duración y severidad de la mucositis en pacientes sometidos a irradiación corporal total y quimioterapia a altas dosis9. La clorhexidina no debe utilizarse, pues no es útil y puede empeorar los síntomas.

La amifostina $\left(\right.$ Ethyo $^{\circledR}{ }^{\circledR}$ ), radioprotector selectivo de los tejidos $\operatorname{sanos}^{10}$ y citoprotector antirradicales libres generados por la radiación ionizante o por agentes quimioterápicos ${ }^{11}$, administrado de forma i.v. no ha sido eficaz en prevenir lá 
mucositis en un estudio fase III con 301 pacientes a una dosis de $200 \mathrm{mgr} / \mathrm{m}^{2}{ }^{12}$. En otro estudio aleatorizado con $50 \mathrm{pa}$ cientes si lo fue a una dosis de $300 \mathrm{mgrs} / \mathrm{m}^{2}{ }^{13}$. Queda por tanto pendiente definir la dosis adecuada.

En cuanto al tratamiento, para el dolor inicialmente se recomienda utilizar AINEs y posteriormente añadir opiáceos. En demostrar la utilidad de los corticoides, destaca un estudio $^{14}$ con enjuagues con betametasona diluida. Las soluciones anestésicas también alivian las molestias en cavidad oral y orofaringe. En nuestro centro empleamos como solución magistral, en 1 litro de agua 3 ampollas de betametasona $\left(\right.$ Celestone ${ }^{\circledR}$ ), 1 ampolla de $10 \mathrm{ml}$ de mepivacaína 2\% (Scandinibsa $\left.{ }^{\circledR}\right), 1$ cucharadita de sal y bicarbonato.

La orgoteína (Ontosein ${ }^{\circledR}$ ) o superóxido dismutasa con $\mathrm{Cu} / \mathrm{Zn}$, es capaz de neutralizar el principal radical libre producido por la interacción de las radiaciones ionizantes con el oxígeno en los tejidos, el anión superóxido $\left(02^{-}\right)$. Dichos radicales libres estan implicados en la eficacia y en la toxicidad de la radioterapia. En los pocos ensayos que existen, se demuestra eficaz para el tratamiento de la mucositis ${ }^{15}$ sin reducir la eficacia en el control tumoral. La dosis habitual es 4 $8 \mathrm{mgr} / 24-48 \mathrm{~h}$ i.m.

La glutamina (Adamin $\mathrm{G}^{\circledR}$ ) diluida en solución para enjuagues parece beneficiosa ${ }^{16}$, aunque no esta clara la dosis recomendable.

Los factores estimulantes de colonias de granulocitos ${ }^{17}$ no parecen aportar beneficio alguno. Los resultados con estimulantes de colonias de granulocitos y macrófagos son contradictorios aunque un estudio si demostró mejoría de los pacientes $^{18}$.

Ni la pentoxifilina, ni el TGF- $\beta 3$ demostraron utilidad en la prevención y tratamiento de la mucositis. Esta estudiándose la posible eficacia de la IL-11.

\section{Xerostomía}

Su intensidad dependerá del porcentaje de glándulas salivares (parótidas, submandibulares y sublinguales) incluidas en el campo de tratamiento. Habitualmente comienza a notarse en la tercera semana de tratamiento, cuando la saliva se torna viscosa y pegajosa, apareciendo posteriormente la sensación de sequedad. Esta provoca malestar, alteración del gusto y caries dental.

La pérdida de saliva puede ser completa y permanente tras dosis superiores a $35 \mathrm{~Gy}$, aunque puede existir recuperación parcial 6-12 meses después del tratamiento si existen zonas glandulares que recibieran dosis menores de $45 \mathrm{~Gy}^{?}$.

En cuanto a la prevención de la xerostomía, en un importante estudio fase III la amifostina $\left(\right.$ Ethyol $\left.^{\circledR}\right)$ ) demostró conservar de forma significativa el flujo salivar y también una pequeña ventaja significativa en la sensación subjetiva de sequedad $^{12}$. En la última reunión de la ASCO, en 2004, se actualizaron los datos de dicho estudio con 2 años de seguimiento mínimo, manteniéndose los resultados favorables sin empeorar la supervivencia. Dado que la administración i.v. de este fármaco provoca náuseas, vómitos e hipotensión, se está estudiando la eficacia de la vía subcutanea para facilitar su uso clínico ${ }^{19}$

La pilocarpina (Salagen ${ }^{\circledR}$ ) es un fármaco colinérgico parasimpáticomimético que estimula la producción de saliva. Ha demostrado ser eficaz en aliviar la xerostomia post-ra- dioterapia. Para la prevención de la xerostomía, dos pequeños estudios no controlados sugirieron beneficio de la pilocarpina como protector de las glándulas salivares durante el tratamiento radioterápico. El daño radioterápico ocurriría tras la liberación intracelular de gránulos conteniendo enzimas proteolíticas, inhibiendo los fármacos sialogogos dicho daño al reducir el número de gránulos. Así se realizaron 2 grandes ensayos aleatorizados que aunque demostraron que mejoraba el flujo de saliva basal, no hubo diferencia en la sensación subjetiva de xerostomía referida por los pacientes ${ }^{11}$.

La utilización de los nuevos avances tecnológicos, como la planificación tridimensional y la radioterapia con modulación de intensidad, pueden ayudar a prevenir la xerostomía al respetar en lo posible las glándulas salivares.

Para el tratamiento de la xerostomía ya establecida, la pilocarpina ha demostrado su eficacia en dos amplios ensayos aleatorizados, doble ciego, recomendándose una dosis de 5 mgrs. 3 veces al día ${ }^{11}$. En ocasiones el efecto no se alcanza plenamente hasta las 4 semanas de iniciar el tratamiento. Los pacientes que no responden a dicha dosis pueden hacerlo duplicándola hasta $10 \mathrm{mgrs} / 8 \mathrm{~h}$, aunque con mayor riesgo de efectos secundarios (hipersudoración, náuseas, rinitis, mareo, lagrimeo, visión borrosa, cefalea, diarrea). Con el tiempo, los pacientes no se vuelven refractarios al tratamiento, que mantiene su eficacia y puede mantenerse indefinidamente si se tolera bien, ya que su beneficio desaparece al discontinuarlo.

Alivian síntomas los sustitutivos de la saliva con carboximetilcelulosa.

Se han descrito casos aislados de mejoría de la xerostomía tras tratamiento de la osteonecrosis con oxigenoterapia hiperbárica.

\section{Sentido del gusto}

La alteración del gusto comienza a la semana de iniciar el tratamiento, progresando rápidamente entre los 20 y 40 Gy. Se suele recuperar completamente entre 2 y 4 meses después de finalizarlo. Un estudio aleatorizado y controlado demostró que el sulfato de $Z n$ a una dosis de 45 mgrs 3 veces al día conseguía un menor deterioro del gusto durante la radioterapia y más rápida recuperación tras completarla ${ }^{20}$.

\section{Sialoadenitis}

A las 12-24 horas de iniciar el tratamiento, alrededor de un $5 \%$ de los pacientes pueden alarmarse por notar hinchazón ligeramente dolorosa de alguna glándula salivar incluida en el campo irradiado. Habitualmente se resuelve en 24-48 horas, no siendo necesario interrumpir el tratamiento. Debemos tranquilizar al paciente que puede creer se trate de progresión tumoral y prescribir AINEs si fueran precisos.

\section{Edema laríngeo}

Puede persistir durante varios meses hasta 1 año. Como tratamiento se recomienda no forzar la voz, corticoides como la dexametasona y si aparece ulceración y dolor antibioticoterapia con ciprofloxacino. Puede añadirse tratamiento con pentoxifilina (Elorgan ${ }^{\circledR}$, Hemovas ${ }^{\circledR}$ ). 


\section{F. Mendicote León}

\section{Atragantamiento y aspiración}

Aparece sobre todo en pacientes sometidos a laringuectomía supraglótica o por fibrosis post-radioterapia, existiendo riesgo de neumonía. Afecta mucho la calidad de vida de los pacientes, que necesitan adecuada rehabilitación para aprender a tragar ${ }^{21}$, siendo a veces preciso el tratamiento quirúrgico. Si ambos fueran ineficaces debería evitarse la ingesta y colocar sonda nasogástrico o de gastrostomía para aporte nutricional.

\section{Fibrosis. Trismus. Edema cervical}

La radioterapia cervical postquirúrgica conlleva un riesgo elevado de fibrosis tardía cutanea y de tejidos blandos. A menudo es progresiva y difícil de manejar. La hipoxia tisular la favorece, y la inflamación por el tratamiento aumenta la expresión del TNF que activa macrófagos productores de citoquinas fibrogénicas. En la severidad de la fibrosis influye la dosis total, dosis por sesión y el volumen tratado.

Cuando la radioterapia afecta la articulación temporomandibular, los músculos pterigoideos o el masetero puede aparecer trismus por fibrosis local, sobre todos cuando se superan dosis de 60 Gy'. Se recomienda iniciar precozmente terapia física realizando movimientos con dispositivos que fuercen la apertura de la boca, por ejemplo con unas simples pinzas de la ropa o más sofisticados como el Therabite $₫$ que lo consigue más eficazmente. Se han comunicado casos de mejoría del trismus con oxigenoterapia hiperbárica.

El tratamiento de cualquier fibrosis puede intentarse con orgoteina, pues dos estudios con la forma liposomal, no comercializada en nuestro medio, parecen demostrar su utili$\operatorname{dad}^{22,23}$

La pentoxifilina mejora el flujo sanguíneo locorregional, inhibe el TNF, y la IL-1 $\beta$ que aumenta la proliferación de fibroblastos. Sendos estudios publicados en los dos últimos años demuestran benefecio en el tratamiento de la fibrosis por radiación, con o sin vitamina $\mathrm{E}$ asociada ${ }^{24,25}$.

Para el edema cervical se recomiendan ejercicios físicos que contraigan la musculatura y activen el drenaje linfático, medidas posturales durante el sueño y el drenaje linfático manual.

\section{Radionecrosis}

La radionecrosis aparece por la destrucción de los tejidos debido a la devascularización secundaria. Tras la inflamación aguda, la piel y mucosas se adelgazan y atrofian, quedando proclives a la formación de úlceras cuya persistencia se debe a la necrosis de los tejidos blandos'.

Si se afecta el hueso subyacente se habla de osteorradionecrosis. Si esta es severa pueden producirse fracturas patológicas, espontáneamente o por la extracción de piezas dentarias. La extracción selectiva de piezas dentarias en mal estado antes de la radioterapia ayuda a prevenir el riesgo de necrosis. No esta indicada la extracción indiscriminada de todos los dientes, pues puede aumentarlo. Tras finalizar la radioterapia es más arriesgado extraer piezas mandibulares que maxilares. El deterioro dental fuera del campo de tratamiento no predispone a la osteonecrosis pues el hueso no recibió dosis altas de radioterapia'.
El NCl recomienda el tratamiento con oxigenoterapia hiperbárica $(\mathrm{OHB})$ antes y después de la extracción de piezas mandibulares de zonas sometidas a dosis altas de radioterapia para prevenir la osteorradionecrosis ${ }^{26}$.

En cuanto al tratamiento, la mayoría de los casos leves o moderados se resuelven espontáneamente con tratamiento conservador, aconsejándose el uso de antisépticos, antibióti$\cos$ (claritromicina, ciprofloxacino, amoxicilina/clavulánico) y evitar el uso de prótesis dentales. El dolor se suele controlar con AINEs y anestésicos tópicos como la lidocaina viscosa. Puede realizarse desbridamiento para facilitar la cicatrización.

Cuando la lesión progresa o provoca dolor intenso debe emplearse inicialmente $\mathrm{OHB}$, y si no es eficaz, cirugía radical (hemimandibulectomía). La OHB consigue una mayor carga de oxígeno diluido en la sangre, aumenta la capacidad de difusión de oxígeno en los tejidos hipóxicos y favorece la angiogénesis ${ }^{27}$.

La condronecrosis es una complicación rara que a menudo comienza por la ulceración de los tejidos blandos que cubren el cartílago. Puede provocarse por la realización de una biopsia, a evitar si no es clara la sospecha de recidiva, aunque los síntomas de la condronecrosis laríngea (disfonía, disfagia, disnea) son similares a los de aquella ${ }^{1}$, siendo preciso realizar a veces traqueostomía. Se recomienda pautar antibióticoterapia, sobre todo el ciprofloxacino a una dosis de $750 \mathrm{mgr} / 12 \mathrm{~h}$, eficaz contra el s. aureus y la pseudomona, y tratamiento esteroideo. Si en 10 días no se aprecia mejoría debe realizarse desbridamiento del tejido necrótico. En ocasiones es precisa la laringuectomía total aunque debe intentarse el tratamiento con $O H B$.

La necrosis de tejidos blandos, se manifiesta por la persistencia de una úlcera sin evidencia de tumor. Puede mejorar con pentoxifilina ${ }^{28}$. Si fracasa valorar tratamiento con $\mathrm{OHB}$ o con arginina (Arginaid ${ }^{\circledR}$ ), precursor del óxido nítrico, vasodilatador, eficaz para el tratamiento de úlceras crónicas en extremidades de otras etiologías ${ }^{29}$.

\section{Estenosis faríngea. Fístulas faringocutáneas}

La estenosis faringea es una complicación rara por fibrosis progresiva tras dosis elevadas de radioterapia, que produce disfagia severa y riesgo de aspiración. Debe proponerse tratamiento con orgoteína, OHB y si fracasan finalmente reconstrucción con injerto pediculado.

Tras laringuectomía más del 15\% de los pacientes presentan fístulas faringocutaneas. Debemos esperar a iniciar el tratamiento hasta que reduzcan su tamaño. La radioterapia previa a la cirugía aumenta el riesgo de dichas fístulas. El tratamiento debe hacerse con antibióticos, evitar la ingesta con sonda nasogástrica y recomendar también no tragar saliva, adecuado drenaje y curas con gasas. La reconstrucción con colgajo se realizará cuando no cierre espontáneamente.

\section{Aparato visual}

La irradiación del polo anterior del ojo y anejos produce efectos agudos como la dermatitis del párpado, conjuntivitis, edema y atrofia de epitelio corneal. El tratamiento debe hacerse con lágrima artificial, colirio antibiótico para prevenir la infeccion, antiinflamatorio (Voltaren colirio ${ }^{\circledR}$ ) ○ corticoideo. 
Los efectos tardíos sobre los párpados incluyen el entropión y ectropion, cuyo tratamiento es quirúrgico, y la estenosis del conducto lacrimal que debe canalizarse para evitar epífora. El daño de las glándulas lacrimales produce el síndrome del ojo seco con queratitis secundaria lo que precisa tratamiento intensivo con lubricantes, parche ocular y colirio antibiótico, para prevenir erosiones corneales ${ }^{30}$.

El riesgo de catarata depende de la dosis total y del fraccionamiento. El cristalino del adulto puede tolerar una dosis total de 5 Gy con fraccionamiento habitual. El 5\% desarrollarán catarata madura durante los 5 años siguientes a recibir una dosis de 10 Gy, y el 50\% con una dosis de 15-18 $\mathrm{Gy}^{31}$, precisando tratamiento quirúrgico. Cuanto mayor sea la dosis, antes se formará la catarata. Se debe intentar prevenir su formación con adecuada protección mediante lentillas plomadas.

El riesgo de retinopatía se relaciona con dosis superiores a $50 \mathrm{~Gy}$, sobre todo cuando se incluye más del $50 \%$ del área de la retina ${ }^{32}$. La aparición de síntomas, pérdida de agudeza visual, de la visión central o aparición de manchas flotantes, oscila entre 18 meses y 5 años. Algunos pacientes también desarrollan glaucoma. El tratamiento mediante fotocoagulación con laser a veces consigue que desaparezca la neovascularización fibrovascular ${ }^{30}$.

La neuropatía óptica por radiación suele presentarse como una pérdida de visión repentina e indolora, monocular. Puede manifestarse también con escotomas centrales $u$ otros defectos campimétricos ${ }^{30}$. No existe tratamiento eficaz por lo que la prevención, evitando llegar a dosis de 54 Gy con fraccionamiento convencional, guardando distancia de seguridad en la radiocirugía estereotáxica y evitando esquemas de hipofraccionamiento que incluyan el nervio óptico, es lo más importante.

\section{Oído}

La inflamación aguda del oido medio y externo puede provocar dolor, hipoacusia y a veces supuración por infección. Se utilizarán descongestionantes, antihistamínicos y vasoconstrictores, como la fenilefrina (en combinados anticatarrales) o la pseudoefedrina con antihistamínicos (Rino-ebaste $\left.{ }^{\circledR}\right)$ y esteroides. Si se sospecha infección, colirio ótico con ciprofloxacino (Baycip ${ }^{\circledR}$ Otico) o bien con neomicina y polimixina B (Synalar ${ }^{\circledR}$ Otico) ${ }^{1}$. A veces es preciso realizar una miringotomía para aliviar los síntomas.

\section{Tiroides. Hipófisis}

La radioterapia cervical produce una alta incidencia, de hasta el 50\%, de hipotiroidismo primario subclínico años después del tratamiento. Sólo el 5\% desarrollan clínica, más frecuente en mujeres que en hombres'. El tratamiento consiste en administrar hormona tiroidea (Levothroid $\left.{ }^{\circledR}\right)$.

El hipotiroidismo puede ser también secundario a la disminución de la TSH hipofisaria por lesión radioterápica de la glándula pituitaria ${ }^{30}$. La del niño es más sensible que la del adulto por lo que deberá vigilarse estrechamente un posible hipopituitarismo para reponer a tiempo la hormona deficitaria.

\section{Bibliografía}

1. Parsons JT. The Effect of Radiation on Normal Tissues of the Head and Neck. En: Million RR, Cassisi NJ, eds. Management of Head and Neck Cancer: A Multidisciplinary Approach, second edition. J.B. Lippincott Company, Philadelphia, 1994, 245-289.

2. Garden AS. Mucositis: Current Management and Investigations. Semin Radiat Oncol 2003, 13: 267-273.

3. Kwong KKF. Prevention and Treatment of Oropharyngeal Mucositis Following Cancer Therapy: Are There New Approaches? Cancer Nurs 2004, 27: 183-205.

4. Köstler WJ, Hejna M, Wenzel C, Zielinski CC. Oral Mucositis Complicating Chemotherapy and/or Radiotherapy: Options for Prevention and Treatment. CA Cancer J Clin 2001, 51: 290315.

5. El-Sayed S, Nabid A, Shelley W et al. Prophylaxis of radiationassociated mucositis in conventionally treated patients with head and neck cancer: a double-blind, phase III, randomized, controlled trial evaluating the clinical efficacy of an antimicrobial lozenge using a validated mucositis scoring system. J Clin Oncol 2002, 20: 3956-63

6. Carl W, Emrich LS. Management of oral mucositis during local radiation and systemic chemotherapy: a study of 98 patients. J Prosthet Dent 1991, 66: 361-369.

7. Ertekin MV, Koç M, Karslio_lu I, Sezen O. Zinc sulfate in the prevention of radiation-induced oropharyngeal mucositis: a prospective, placebo-controlled, randomized study. Int J Radiat Oncol Biol Phys 2004, 58: 167-174.

8. Rubenstein $E B$, Peterson $D E$, Schubert $M$, et al. Clinical Practice Guidelines for the Prevention and Treatment of Cancer TherapyInduced Oral and Gastrointestinal Mucositis. Cancer 2004, 100 (9 suppl): 2026-2046.

9. Spielberger R, Stiff P, Bensinger W, et al. Palifermin for Oral Mucositis after Intensive Therapy for Hematologic Cancers. N Engl J Med 2004, 351: 2590-8.

10. Koukourakis MI. Amifostine: Is There Evidence of Tumor Protection? Semin Oncol 2003, 30: 18-30.

11. Eisbruch A, Rhodus N, Rosenthal D, et al. The Prevention and Treatment of Radiotherapy-Induced Xerostomia. Semin Radiat Oncol 2003, 13: 302-308.

12. Brizel DM, Wasserman TH, Henke M, et al. Phase III Randomized Trial of Amifostine as a Radioprotector in Head and Neck Cancer. J Clin Oncol 2000; 18: 3339-3345.

13. Antonadou $D$, Pepelassi $M$, Synodinou $M$, et al. Prophylactic use of amifostine to prevent radiochemotherapy-induced mucositis and xerostomia in head-and-neck cancer. Int J Radiat Oncol Biol Phys 2002, 52: 739-747.

14. Aldelaal AS, Barker DS, Fergusson MM. Treatment of irradiation-induced mucositis. Lancet 1989, 333: 97.

15. Valencia J, Velilla C, Urpegui A et al.The efficacy of orgotein in the treatment of acute toxicity due to radiotherapy on head and neck tumors. Tumori 2002: 385-389.

16. Huang E-Y, Leung SW, Wang C-J, et al. Oral glutamine to alleviate radiation-induced oral mucositis: a pilot randomized trial. Int J Radiat Oncol Biol Phys 2000, 46: 535-539.

17. Su YB, Vickers A, Zelefsky MJ, et al. Double-blind, randomized trial of granulocyte-colony stimulating factor (GCSF) versus placebo during postoperative radiation (RT) for advanced resectable squamous cell head and neck cancer (SCCHN): Impact on mucositis. J Clin Oncol 2004, 22 (14S): 5587 (ASCO Annual Meeting Proceedings). 


\section{F. Mendicote León}

18. Wagner W, Alfrink M, Haus U, Matt J. Treatment of irradiation-induced mucositis with growth factors (rhGM-CSF) in patients with head and neck cancer. Anticancer Res 1999, 19: 799-803.

19. Bardet E, Martin L, Calais G, et al. Preliminary Data of the GORTEC 2000-02 Phase III Trial Comparing Intravenous and Subcutaneous Administration of Amifostine for Head and Neck Tumors Treated by External Radiotherapy. Semin Oncol 2002, 29 (suppl 19): 57-60.

20. Ripamonti C, Zecca E, Brunelli $C$, et al. A randomized, controlled clinical trial to evaluate the effects of zinc sulfate on cancer patients with taste alterations caused by head and neck irradiation. Cancer 1998, 82:1938-1945.

21. Mittal BB, Pauloski BR, Haraf DJ, et al. Swallowing dysfunctionpreventative and rehabilitation strategies in patients with headand-neck cancers treated with surgery, radiotherapy, and chemotherapy: a critical review. Int J Radiat Oncol Biol Phys 2003, 57:1219-1230.

22. Delanian $S$, Baillet $F$, Huart J, et al. Successful treatment of radiation-induced fibrosis using liposomal $\mathrm{Cu} / \mathrm{Zn}$ superoxide dismutase: clinical trial. Radiother Oncol 1994, 32: 12-20.

23. Lefaix JL, Delanian $S$, Leplat JJ, et al. Successful treatment of radiation-induced fibrosis using Cu/Zn-SOD and Mn-SOD: An experimental study. Int J Radiat Oncol Biol Phys 1996, 35: 305-312.

24. Delanian S, Porcher R, Balla-Mekias S, Lefaix JL. Randomized, Placebo-Controlled Trial of Combined Pentoxifylline and Tocopherol for Regresion of Superficial Radiation-Induced Fibrosis. J Clin Oncol 2003, 21: 2545-2550
25. Okunieff $P$, Augustine E, Hicks JE et al. Pentoxifylline in the Treatment of Radiation-Induced Fibrosis. J Clin Oncol 2004, 22: 2207-2213.

26. Neumeister M. Hyperbaric Oxygen Therapy. www.emedicine. com 2004 November 11

27. Stone HB, McBride WH, Coleman CN. Modifying Normal Tissue Damage Postirradiation Report of a Workshop Sponsored by the Radiation Research Program, National Cancer Institute, Bethesda, Maryland September 6-8, 2000. Radiat Res 2002, 157: 204-223

28. Moulder JE. Pharmacological Intervention to Prevent or Ameliorate Chronic Radiation Injuries. Semin Radiat Oncol 2003, 13: 73-84.

29. Eremin $O$ ed. L-Arginine: Biological Aspects and Clinical Applications. R.G. Landes Company, Springer, 1997.

30. Nicolaou N. Prevention and management of radiation toxicity. En: Pazdur R, Hoskins WJ, Wagman L, Coia LR eds. Cancer Management: A Multidisciplinary Approach. Publisher Research \& Representation, Inc, 2004, 927-957.

31. Henk JM, Whitelocke RA, Warrington AP, Bessell EM. Radiation dose to the lens and cataract formation. Int J Radiat Oncol Biol Phys 1993, 25: 815-820.

32. Takeda A, Shigematsu N, Suzuki S, et al. Late retinal complications of radiation therapy for nasal and paranasal malignancies: relationship between irradiated-dose area and severity. Int J Radiat Oncol Biol Phys 1999, 44: 599-605. 